Nonlinear Processes in Geophysics (2005) 12: 55-66

SRef-ID: $1607-7946 / \mathrm{npg} / 2005-12-55$

European Geosciences Union

(C) 2005 Author(s). This work is licensed

under a Creative Commons License.

\title{
Testing and modelling autoregressive conditional heteroskedasticity of streamflow processes
}

\author{
W. Wang ${ }^{1,2}$, P. H. A. J. M. Van Gelder ${ }^{2}$, J. K. Vrijling ${ }^{2}$, and J. $\mathrm{Ma}^{3}$ \\ ${ }^{1}$ Faculty of Water Resources and Environment, Hohai University, Nanjing, 210098, China \\ ${ }^{2}$ Faculty of Civil Engineering \& Geosciences, Section of Hydraulic Engineering, Delft University of Technology, P.O.Box \\ 5048, 2600 GA Delft, The Netherlands \\ ${ }^{3}$ Yellow River Conservancy Commission, Hydrology Bureau, Zhengzhou, 450004, China
}

Received: 24 May 2004 - Revised: 15 December 2004 - Accepted: 5 January 2005 - Published: 21 January 2005

Part of Special Issue "Nonlinear deterministic dynamics in hydrologic systems: present activities and future challenges"

\begin{abstract}
Conventional streamflow models operate under the assumption of constant variance or season-dependent variances (e.g. ARMA (AutoRegressive Moving Average) models for deseasonalized streamflow series and PARMA (Periodic AutoRegressive Moving Average) models for seasonal streamflow series). However, with McLeod-Li test and Engle's Lagrange Multiplier test, clear evidences are found for the existence of autoregressive conditional heteroskedasticity (i.e. the ARCH (AutoRegressive Conditional Heteroskedasticity) effect), a nonlinear phenomenon of the variance behaviour, in the residual series from linear models fitted to daily and monthly streamflow processes of the upper Yellow River, China. It is shown that the major cause of the ARCH effect is the seasonal variation in variance of the residual series. However, while the seasonal variation in variance can fully explain the ARCH effect for monthly streamflow, it is only a partial explanation for daily flow. It is also shown that while the periodic autoregressive moving average model is adequate in modelling monthly flows, no model is adequate in modelling daily streamflow processes because none of the conventional time series models takes the seasonal variation in variance, as well as the ARCH effect in the residuals, into account. Therefore, an ARMA-GARCH (Generalized AutoRegressive Conditional Heteroskedasticity) error model is proposed to capture the $\mathrm{ARCH}$ effect present in daily streamflow series, as well as to preserve seasonal variation in variance in the residuals. The ARMA-GARCH error model combines an ARMA model for modelling the mean behaviour and a GARCH model for modelling the variance behaviour of the residuals from the ARMA model. Since the GARCH model is not followed widely in statistical hydrology, the work can be a useful ad-

Correspondence to: W. Wang

(w.wang@126.com)
\end{abstract}

dition in terms of statistical modelling of daily streamflow processes for the hydrological community.

\section{Introduction to autoregressive conditional het- eroskedasticity}

When modelling hydrologic time series, we usually focus on modelling and predicting the mean behaviour, or the first order moments, and are rarely concerned with the conditional variance, or their second order moments, although unconditional season-dependent variances are usually considered. The increased importance played by risk and uncertainty considerations in water resources management and flood control practice, as well as in modern hydrology theory, however, has necessitated the development of new time series techniques that allow for the modelling of time varying variances.

ARCH-type models, which originate from econometrics, give us an appropriate framework for studying this problem. Volatility (i.e. time-varying variance) clustering, in which large changes tend to follow large changes, and small changes tend to follow small changes, has been well recognized in financial time series. This phenomenon is called conditional heteroskedasticity, and can be modeled by ARCH-type models, including the ARCH model proposed by Engle (1982) and the later extension GARCH (generalized ARCH) model proposed by Bollerslev (1986), etc. Accordingly, when a time series exhibits autoregressive conditionally heteroskedasticity, we say it has the ARCH effect or GARCH effect. ARCH-type models have been widely used to model the ARCH effect for economic and financial time series.

The ARCH-type model is a nonlinear model that includes past variances in the explanation of future variances. ARCH- 




Fig. 1. Daily streamflow $\left(\mathrm{m}^{3} / \mathrm{s}\right)$ of the upper Yellow River at Tangnaihai.

type models can generate accurate forecasts of future volatility, especially over short horizons, therefore providing a better estimate of the forecast uncertainty which is valuable for water resource management and flood control. And they take into account excess kurtosis (i.e. fat tail behaviour), which is common in hydrologic processes. Therefore, ARCHtype models could be very useful for hydrologic time series modelling. Some authors propose new models to reproduce the asymmetric periodic behaviour with large fluctuations around large streamflow and small fluctuations around small streamflow (e.g. Livina et al., 2003), which basically can be handled with those conventional time series models that have taken season-dependent variance into account, such as PARMA models and deseasonalized ARMA models. However, little attention has been paid so far by the hydrologic community to test and model the possible presence of the ARCH effect with which large fluctuations tend to follow large fluctuations, and small fluctuations tend to follow small fluctuations in streamflow series.

In this paper, we will take the daily and monthly streamflow of the upper Yellow River at Tangnaihai in China as case study hydrologic time series to test for the existence of the ARCH effect, and propose an ARMA-GARCH error model for daily flow series. The paper is organized as follows. First, the method of testing conditional heteroskedasticity of streamflow process is described. Then, the causes of the ARCH effect and the inadequacy of commonly used seasonal time series models for modelling streamflow are discussed. Finally, an ARMA-GARCH error model is proposed for capturing the ARCH effect existing in daily streamflow series.

\section{Case study area and data set}

The case study area is the headwaters of the Yellow River, located in the northeastern Tibet Plateau. In this area, the discharge gauging station Tangnaihai has a $133650 \mathrm{~km}^{2}$ con-

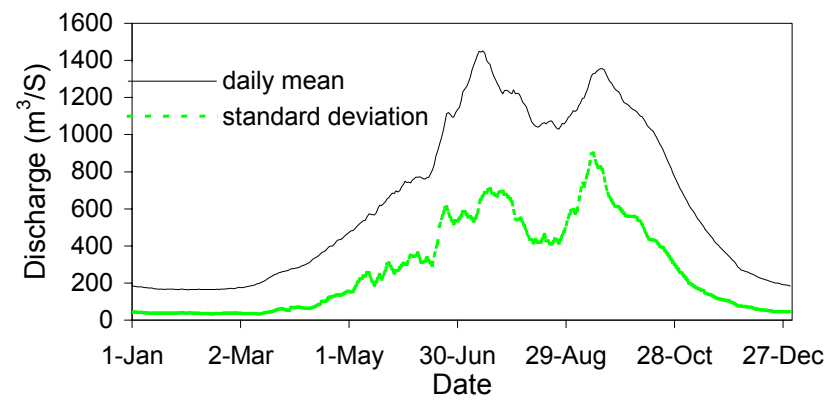

Fig. 2. Variation in daily mean and standard deviation of the streamflow at Tangnaihai.

tributing watershed, including a permanently snow-covered area of $192 \mathrm{~km}^{2}$. The length of the main channel of this watershed is over $1500 \mathrm{~km}$. Most of the area is $3000 \sim 6000$ meters above sea level. Snowmelt water composes about 5\% of total runoff. Most rain falls in summer. Because the watershed is partly permanently snow-covered and sparsely populated, without any large-scale hydraulic works, it is fairly pristine. The average annual runoff volume (during 19562000) at Tangnaihai gauging station is 20.4 billion cubic meters, about $35 \%$ of the whole Yellow River Basin, and it is the major runoff producing area of the Yellow River basin. Daily average streamflow at Tangnaihai has been recorded since 1 January 1956. Monthly series is obtained from daily data by taking the average of daily discharges in every month. In this study, data from 1 January 1956 to 31 December 2000 are used. The daily streamflow series from 1956 to 2000 is plotted in Fig. 1, and variations in the daily mean discharge and daily standard deviation of the streamflow at Tangnaihai are shown in Fig. 2.

\section{Tests for the ARCH effect of streamflow process}

The detection of the ARCH effect in a streamflow series is actually a test of serial independence applied to the serially uncorrelated fitting error of some model, usually a linear autoregressive (AR) model. We assume that linear serial dependence inside the original series is removed with a well-fitted, pre-whitening model; any remaining serial dependence must be due to some nonlinear generating mechanism which is not captured by the model. Here, the nonlinear mechanism we are concerned with is the conditional heteroskedasticity. We will show that the nonlinear mechanism remaining in the pre-whitened streamflow series, namely the residual series, can be well interpreted as autoregressive conditional heteroskedasticity.

\subsection{Linear ARMA models fitted to daily and monthly flows}

Three types of seasonal time series models are commonly used to model hydrologic processes which usually have strong seasonality (Hipel and McLeod, 1994): 1) seasonal autoregressive integrated moving average (SARIMA) mod- 

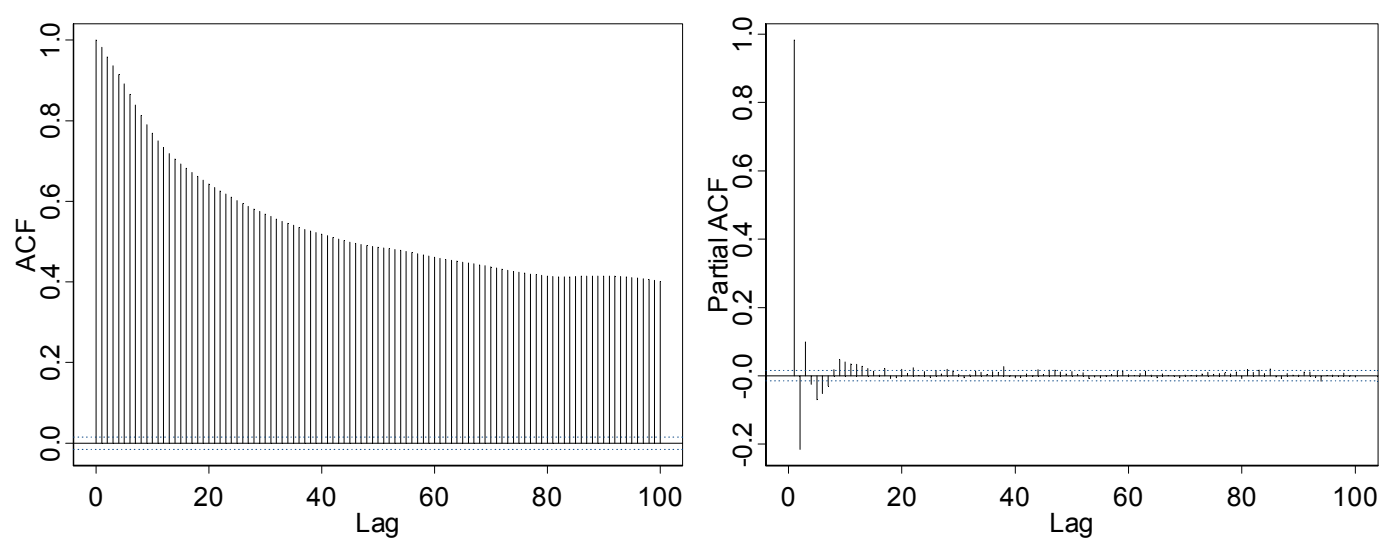

Fig. 3. ACF and PACF of deseasonalized daily flow series.
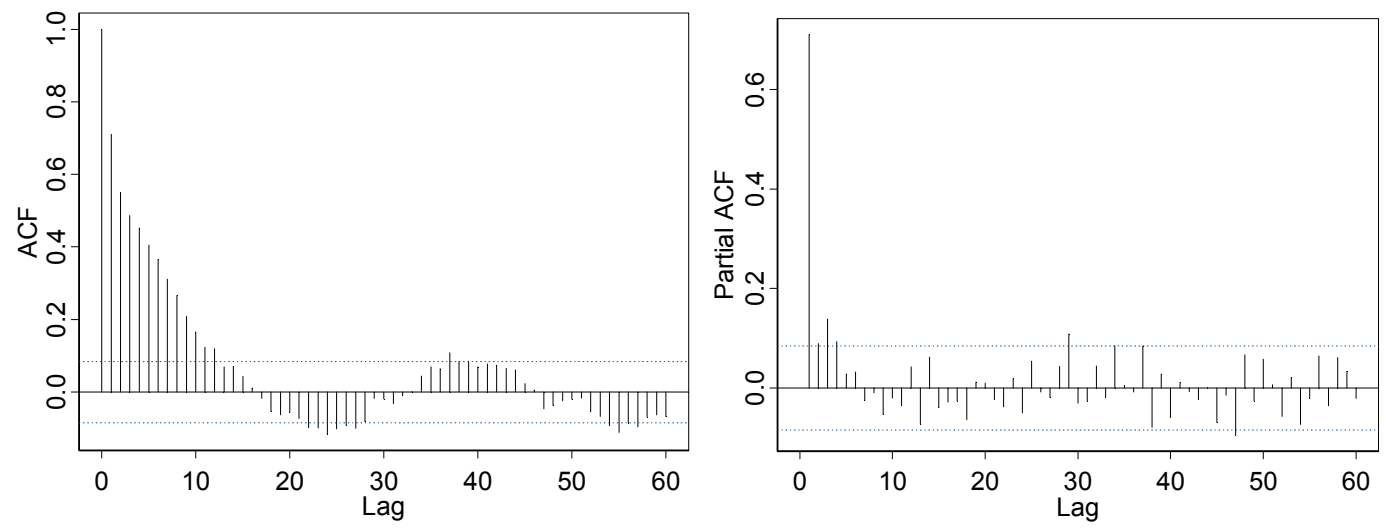

Fig. 4. ACF and PACF of deseasonalized monthly flow series.

els; 2) deseasonalized ARMA models; and 3) periodic ARMA models. The deseasonalized modelling approach is adopted in this study. The procedure of fitting deseasonalized ARMA models to daily and monthly streamflow at Tangnaihai includes two steps. First, logarithmize both flow series, and deseasonalize them by subtracting the seasonal (e.g. daily or monthly) mean values and dividing by the seasonal standard deviations of the logarithmized series. To alleviate the stochastic fluctuations of the daily means and standard deviations, we smooth them with first 8 Fourier harmonics before using them for standardization. Then, according to the ACF (AutoCorrelation Function) and PACF (Periodic AutoCorrelation Function) structures of the two series, as well as the model selection criterion AIC, two linear ARMA-type models (one $\operatorname{ARMA}(20,1)$ and one $\operatorname{AR}(4))$ are fitted to the logarithmized and deseasonalized daily and monthly flow series, respectively, following the model construction procedures suggested by Box and Jenkins (1976). Figures 3 and 4 show the ACF and PACF of the deseasonalized daily and monthly series. Figure 5 shows parts of the two residual series obtained from the two models.

Before applying ARCH tests to the residual series, to ensure that the null hypothesis of no ARCH effect is not rejected due to the failure of the pre-whitening linear models, we must check the goodness-of-fit of the linear models.

Firstly, we inspect the ACF of the residuals. It is wellknown that for random and independent series of length $n$, the lag $k$ autocorrelation coefficient is normally distributed with a mean of zero and a variance of $1 / n$, and the $95 \%$ confidence limits are given by $\pm 1.96 / \sqrt{ } \mathrm{n}$. The ACF plots in Fig. 6 show that there is no significant autocorrelation left in the residuals from both ARMA-type models for daily and monthly flow.

Then, more formally, we apply the Ljung-Box test (Ljung and Box, 1978) to the residual series, which tests whether the first $L$ autocorrelations $\hat{r}_{k}^{2}\left(\varepsilon^{2}\right)(k=1, \ldots, L)$ from a process are collectively small in magnitude. Suppose we have the first $L$ autocorrelations $\hat{r}_{k}(\varepsilon)(k=1, \ldots, L)$ from any $\operatorname{ARMA}(p, d, q)$ process. For a fixed sufficiently large $L$, the usual Ljung-Box $Q$-statistic is given by

$Q=N(N+2) \sum_{k=1}^{L} \frac{\hat{r}_{k}^{2}(\varepsilon)}{N-k}$,

where $N=$ sample size, $L=$ the number of autocorrelations included in the statistic, and $\hat{r}_{k}^{2}$ is the squared sample autocorrelation of residual series $\left\{\varepsilon_{t}\right\}$ at lag $k$. Under the null hypothesis of model adequacy, the test statistic is asymptotically $\chi^{2}(L-p-q)$ distributed. Thus, we would reject the null hypothesis at level $\alpha$ if the value of $Q$ exceeds the 

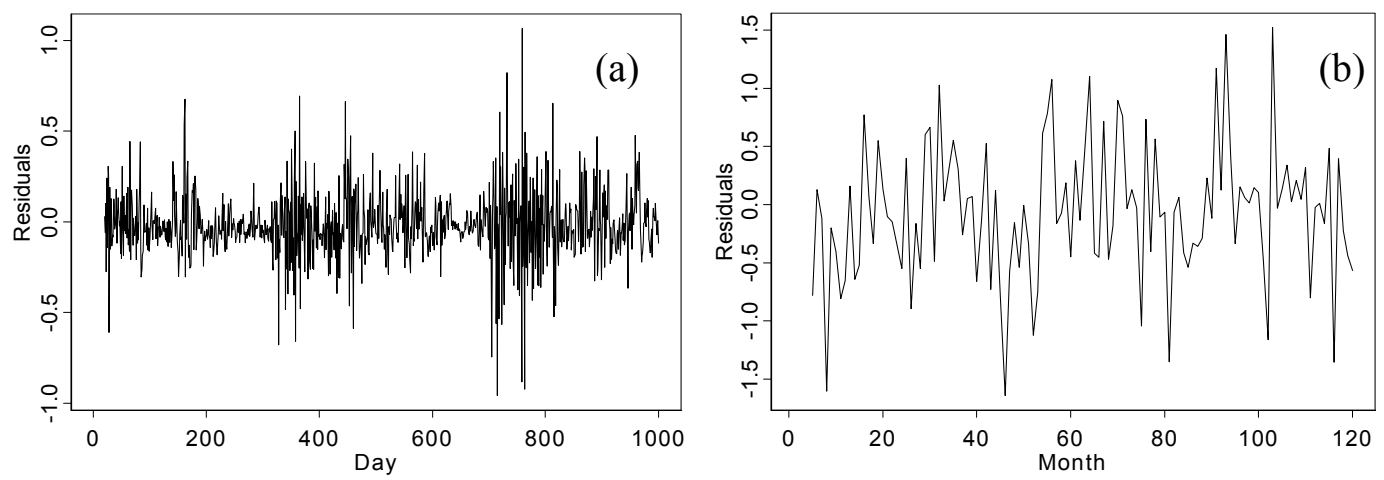

Fig. 5. Segments of the residual series from (a) $\operatorname{ARMA}(20,1)$ for daily flow and (b) AR(4) for monthly flow at Tangnaihai.
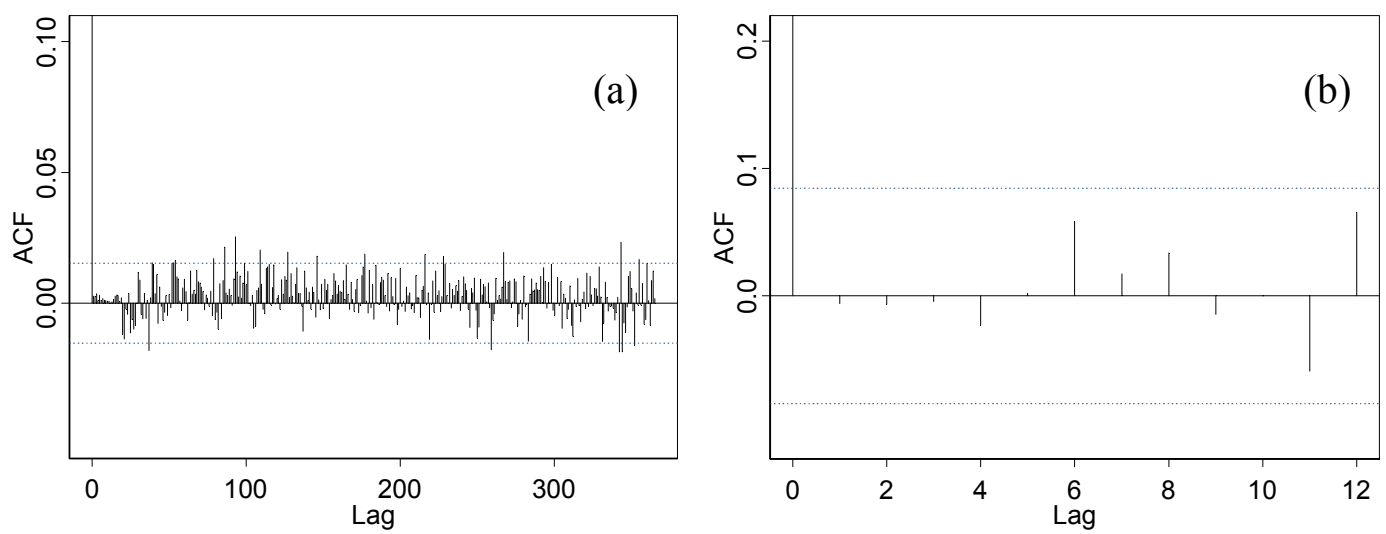

Fig. 6. ACFs of residuals from (a) the $\operatorname{ARMA}(20,1)$ model for daily flow and (b) the AR(4) model for monthly flow at Tangnaihai.

$(1-\alpha)$-quantile of the $\chi^{2}(L-p-q)$ distribution. The LjungBox test results for $\operatorname{ARMA}(20,1)$ and $\operatorname{AR}(4)$ are shown in Fig. 7. The p-values' exceedance of 0.05 indicates the acceptance of the null hypothesis of model adequacy at significance level 0.05 .

However, while the residuals seem statistically uncorrelated according to ACF and PACF shown in Fig. 6, they are not identically distributed from visual inspection of the Fig. 5, that is, the residuals are not independent and identically distributed (i.i.d.) through time. There is a tendency, especially for daily flow, that large (small) absolute values of the residual process are followed by other large (small) values of unpredictable sign, which is a common behaviour of GARCH processes. Granger and Andersen (1978) found that some of the series modelled by Box and Jenkins (1976) exhibit autocorrelated squared residuals even though the residuals themselves do no seem to be correlated over time, and therefore suggested that the ACF of the squared time series could be useful in identifying nonlinear time series. Bollerslev (1986) stated that the ACF and PACF of squared process are useful in identifying and checking GARCH behaviour.

Figure 8 shows the ACFs of the squared residual series from the $\operatorname{ARMA}(20,1)$ model for daily flow and the $\operatorname{AR}(4)$ model for monthly flow at Tangnaihai. It is shown that although the residuals are almost uncorrelated, as shown in
Fig. 6, the squared residual series are autocorrelated, and the ACF structures of both squared residual series exhibit strong seasonality. This indicates that the variance of residual series is conditional on its past history, namely, the residual series may exhibit an ARCH effect.

There are some formal methods to test for the ARCH effect of a process, such as the McLeod-Li test (McLeod and $\mathrm{Li}, 1983$ ), the Engle's Lagrange Multiplier test (Engle, 1982), the BDS test (Brock et. al., 1996), etc. McLeodLi test and Engle's Lagrange Multiplier test are used here to check the existence of an ARCH effect in the streamflow series.

\subsection{McLeod-Li test for the ARCH effect}

McLeod and $\mathrm{Li}$ (1983) proposed a formal test for ARCH effect based on the Ljung-Box test. It looks at the autocorrelation function of the squares of the pre-whitened data, and tests whether the first $L$ autocorrelations for the squared residuals are collectively small in magnitude.

Similar to Eq. (1), for fixed sufficiently large $L$, the LjungBox $Q$-statistic of Mcleod-Li test is given by

$Q=N(N+2) \sum_{k=1}^{L} \frac{\hat{r}_{k}^{2}\left(\varepsilon^{2}\right)}{N-k}$ 
(a)

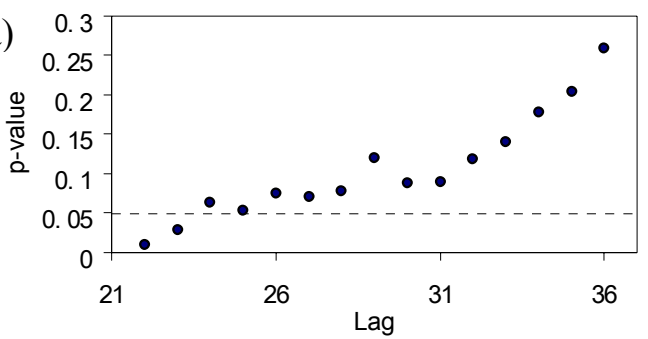

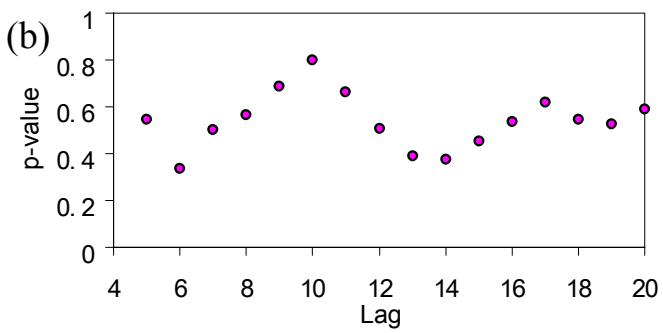

Fig. 7. Ljung-Box lack-of-fit tests for (a) the ARMA(20,1) model for daily flow and (b) the AR(4) model for monthly flow.
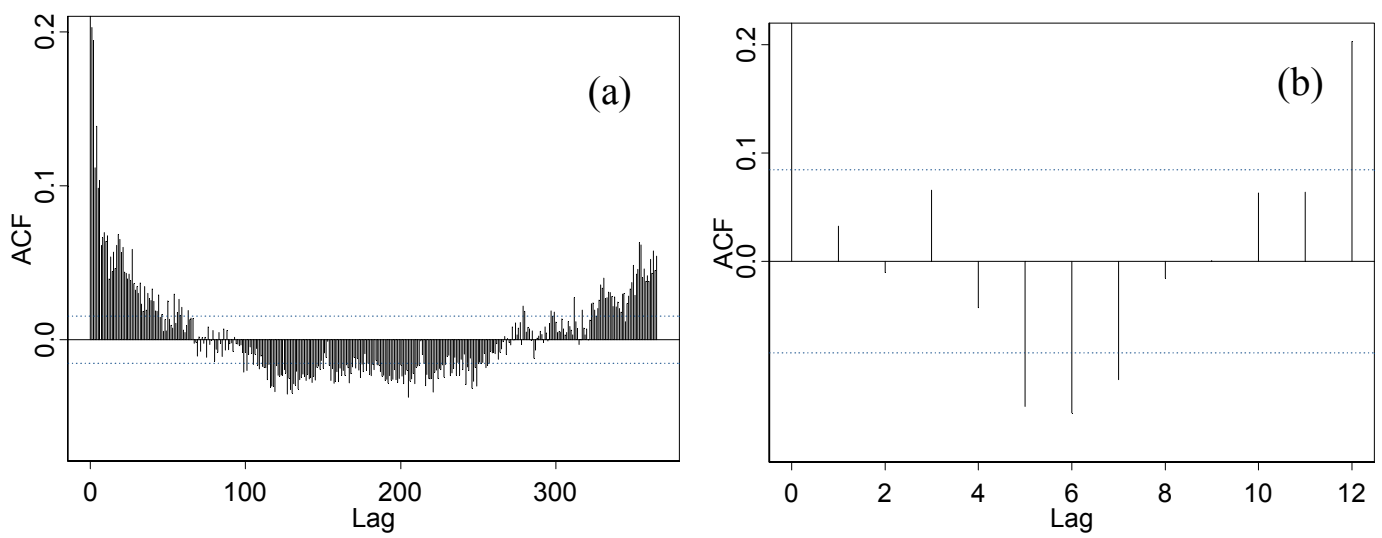

Fig. 8. ACFs of the squared residuals from (a) the ARMA(20,1) model for daily flow and (b) the AR(4) model for monthly flow at Tangnaihai.

where $N$ is the sample size, and $\hat{r}_{k}^{2}$ is the squared sample autocorrelation of squared residual series at lag $k$. Under the null hypothesis of a linear generating mechanism for the data, namely, no ARCH effect in the data, the test statistic is asymptotically $\chi^{2}(L)$ distributed. Figure 9 shows the results of the McLeod-Li test for daily and monthly flow. It illustrates that the null hypothesis of no ARCH effect is rejected for both daily and monthly flow series.

\subsection{Engle's Lagrange Multiplier test for the ARCH effect}

Since the ARCH model has the form of an autoregressive model, Engle (1982) proposed the Lagrange Multiplier (LM) test, in order to test for the existence of ARCH behaviour based on the regression. The test statistic is given by $T R^{2}$, where $R$ is the sample multiple correlation coefficient computed from the regression of $\varepsilon_{t}^{2}$ on a constant and $\varepsilon_{t-1}^{2}, \ldots, \varepsilon_{t-q}^{2}$, and $T$ is the sample size. Under the null hypothesis that there is no ARCH effect, the test statistic is asymptotically distributed as chi-square distribution with $q$ degrees of freedom. As Bollerslev (1986) suggested, it should also have power against GARCH alternatives.

Figure 10 shows Engle's LM test results for the residuals from the $\operatorname{ARMA}(20,1)$ model for daily flow and from the AR(4) model for monthly flow. The results also firmly indicate the existence of an ARCH effect in both the residual series.

One point that should be noticed is that although Figs. 8b, $9 \mathrm{~b}$ and $10 \mathrm{~b}$ show that for monthly flow, autocorrelations at lags less than 4 are removed by the AR(4) model, when we take autocorrelations at longer lags into consideration, significant autocorrelations remain and the null hypothesis of no $\mathrm{ARCH}$ effect is rejected. Because it is required for the McLeod-Li test to use sufficiently large $L$, namely, a sufficient number of autocorrelations to calculate the LjungBox statistic (typically around 20), we still consider that the monthly flow has the ARCH effect.

On the whole, evidences are clear with the McLeod-Li test and Engle's LM test about the existence of conditional heteroskedasticity in the residual series from linear models fitted to the logarithmized and deseasonalized daily and monthly streamflow processes of the upper Yellow River at Tangnaihai.

\section{Discussion of the causes of ARCH effects and inade- quacy of commonly used seasonal time series models}

\subsection{Causes of ARCH effects in the residuals from ARMA-} type models for daily and monthly flow

From the above analyses, it is clear that although the residuals are serially uncorrelated, they are not independent through time. At the mean time, we notice that seasonality dominates autocorrelation structures of squared residual series for both daily and monthly flow processes (as shown in Fig. 8). This suggests that there are seasonal variations in the variance of the residual series, and we should standardize 
(a)

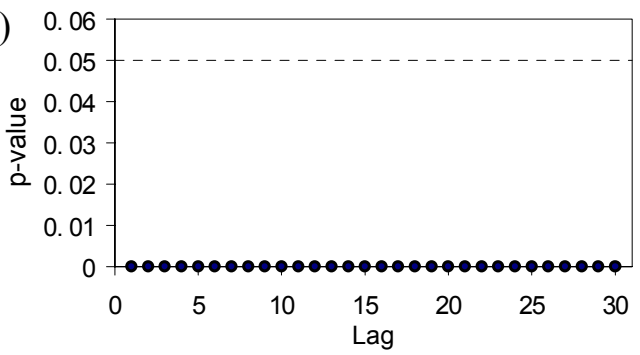

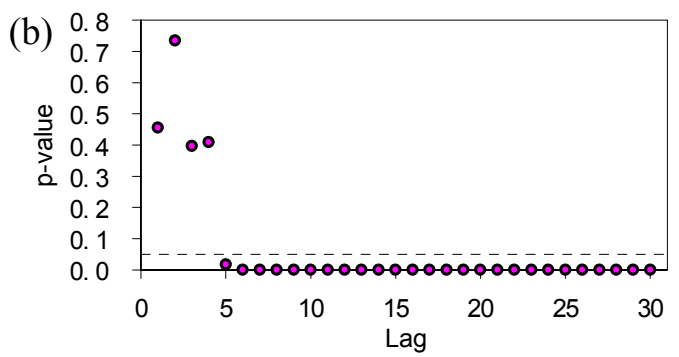

Fig. 9. McLeod-Li test for the residuals from (a) the ARMA(20,1) model for daily flow and (b) the AR(4) model for monthly flow.
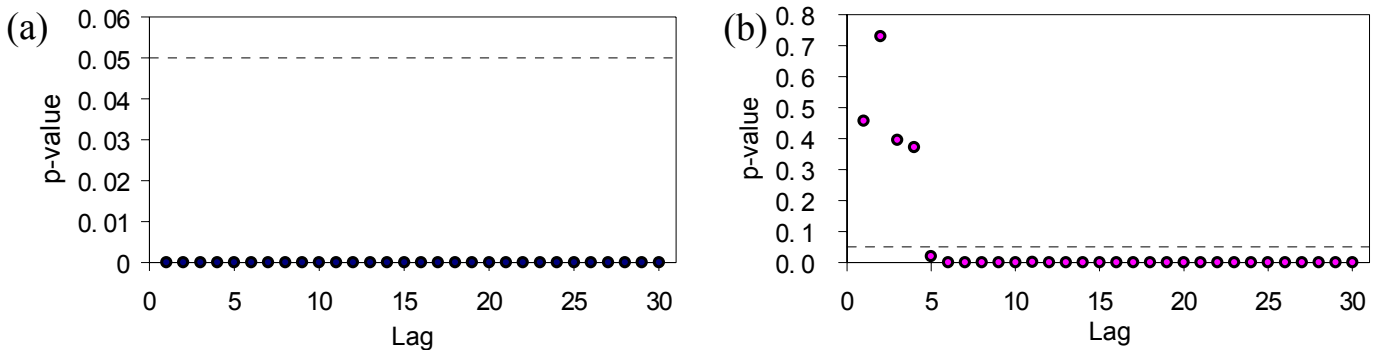

Fig. 10. Engle's LM test for residuals from (a) the ARMA $(20,1)$ model for daily flow and (b) the AR(4) model for monthly flow.

the residual series from linear models with seasonal standard deviations of the residuals first, then look at the standardized series to check whether seasonal variances can explain ARCH effects.

Seasonal standard deviations of the residual series from the $\operatorname{ARMA}(20,1)$ model for daily flow and the AR(4) model for monthly flow are calculated and shown in Figs. 11a and 11b. They are used to standardize the residual series from the $\operatorname{ARMA}(20,1)$ model and the $\operatorname{AR}(4)$ model. Figure 12 shows the ACFs of the squared standardized residual series of daily and monthly flow. It is illustrated that, after seasonal standardized autocorrelation, as well as the seasonality in the squared standardized residual series for monthly flow is basically removed (Fig. 12b), the significant autocorrelation still exists in the squared standardized residual series for daily flow (Fig. 12a), despite the fact that the autocorrelations are significantly reduced compared with Fig. 8a and the seasonality in the ACF structure is removed. This means that the seasonality, as well as the autocorrelation in the squared residuals from the AR model of monthly flow series is basically caused by seasonal variances. But seasonal variances only explain partly the autocorrelation in the squared residuals of daily flow series.

The residual series of daily flow and monthly flow standardized by seasonal standard deviation are also tested for ARCH effects with the McLeod-Li test and Engle's LM test. Figure 13 shows that the seasonally standardized residual series of daily flow still cannot pass the LM test (Fig. 13a), whereas the seasonally standardized residual series of monthly flow pass the LM test with high $p$-values (Fig. 13b). The McLeod-Li test gives similar results.
From the above analyses, it is clear that the ARCH effect is fully caused by seasonal variances for monthly flow, but only partly for daily flow. Other causes, besides the seasonal variation in variance, of the ARCH effect in daily flow may include the perturbations of the temperature fluctuations which is an influential factor for snowmelt, as well as evapotranspiration, and the precipitation variation which is the dominant factor for streamflow processes. As reported by Miller (1979), when modelling a daily average streamflow series, the residuals from a fitted AR(4) model signaled white-noise errors, but the squared residuals signaled bilinearity. When precipitation covariates were included in the model, Miller found that neither the residuals nor the squared residuals signaled any problems. While we agree that the autocorrelation existing in the squared residuals is basically caused by a precipitation process, we want to show that the autocorrelation in the squared residuals can be well described by an $\mathrm{ARCH}$ model, which is very close to the bilinear model (Engle, 1982).

4.2 Inadequacy of commonly used seasonal time series models for modelling streamflow processes

As mentioned in Sect. 3.1, SARIMA models, deseasonalized ARMA models and periodic models are commonly used to model hydrologic processes (Hipel and McLeod, 1994). Given a time series $\left(x_{t}\right)$, the general form of SARIMA model, denoted by $\operatorname{SARIMA}(\mathrm{p}, \mathrm{d}, \mathrm{q}) \times(\mathrm{P}, \mathrm{D}, \mathrm{Q})_{S}$, is

$\phi(B) \Phi\left(B^{s}\right) \nabla^{d} \nabla_{s}^{D} x_{t}=\theta(B) \Theta\left(B^{s}\right) \varepsilon_{t}$,

where $\phi(B)$ and $\theta(B)$ of orders $p$ and $q$ represent the ordinary autoregressive and moving average components; $\Phi\left(B^{S}\right)$ 
(a)

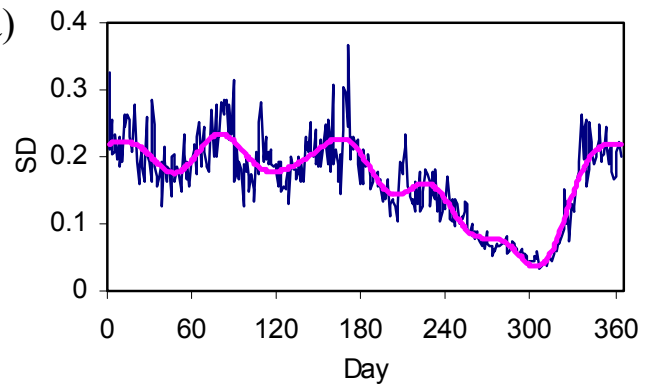

(b)

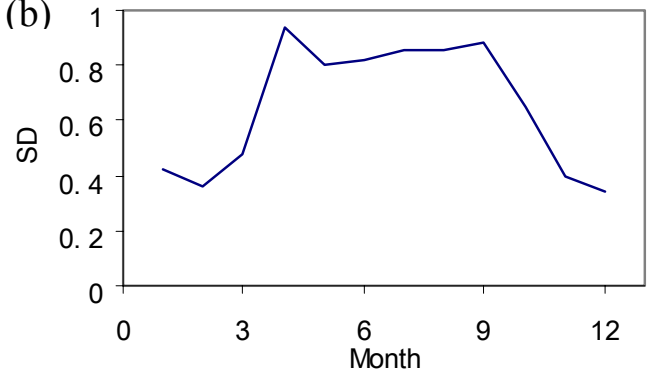

Fig. 11. Seasonal standard deviations (SD) of the residuals from (a) the ARMA $(20,1)$ model for daily flow and (b) the AR(4) model for monthly flow (note: the smoothed line in (a) is given by the first 8 Fourier harmonics of the seasonal SD series).


Fig. 12. ACFs of squared seasonally standardized residuals from (a) the ARMA $(20,1)$ model for daily flow and (b) the AR(4) model for monthly flow.

and $\Theta\left(B^{s}\right)$ of orders $P$ and $Q$ represent the seasonal autoregressive and moving average components; $\nabla^{d}=(1-B)^{d}$ and $\nabla_{S}^{D}=\left(1-B^{S}\right)^{D}$ are the ordinary and seasonal difference components.

The general form of the $\operatorname{ARMA}(p, q)$ model fitted to deseasonalized series is

$\phi(B) x_{t}=\theta(B) \varepsilon_{t}$.

From the model equations we know that although the seasonal variation in the variance present in the original time series is basically dealt with well by the deseasonalized approach, the seasonal variation in variance in the residual series is not considered by either of the two models, because in both cases the innovation series $\varepsilon_{t}$ is assumed to be i.i.d. $N(0$, $\sigma^{2}$ ). Therefore, both SARIMA models and deseasonalized models cannot capture the ARCH effect that we observed in the residual series.

In contrast, the periodic model, which is basically a group of ARMA models fitted to separate seasons, allows for seasonal variances in not only the original series but also the residual series. Taking the special case $\operatorname{PAR}(p)$ model (periodic autoregressive model of order $p$ ) as an example of a PARMA model, given a hydrological time series $x_{n, s}$, in which $n$ defines the year and $s$ defines the season (could rep- resent a day, week, month or season), we have the following $\operatorname{PAR}(p)$ model (Salas, 1993):

$x_{n, s}=\mu_{s}+\sum_{j=1}^{p} \phi_{j, s}\left(x_{v, s-j}-\mu_{s-j}\right)+\varepsilon_{n, s}$,

where $\varepsilon_{n, s}$ is an uncorrelated normal variable with mean zero and variance $\sigma_{s}^{2}$. For daily streamflow series, to make the model parsimonious, we can cluster the days in the year into several groups and fit separate AR models to separate groups (Wang et al., 2004). Periodic models would perform better than the SARIMA model and the deseasonalized ARMA model for capturing the ARCH effect, because it takes season-varying variances into account. However, as analyzed in Sect. 4.1, while considering seasonal variances could be sufficient for describing the ARCH effect in monthly flow series because the ARCH effect in monthly flow series is fully caused by seasonal variances, it is still insufficient to fully capture the ARCH effect in daily flow series.

In summary, while the PARMA model is adequate for modelling the variance behaviour for monthly flow, none of the commonly used seasonal models is efficient enough to describe the ARCH effect for daily flow, although PARMA can partly describe it by considering seasonal variances. It is necessary to apply the GARCH model to achieve the purpose. 
(a)

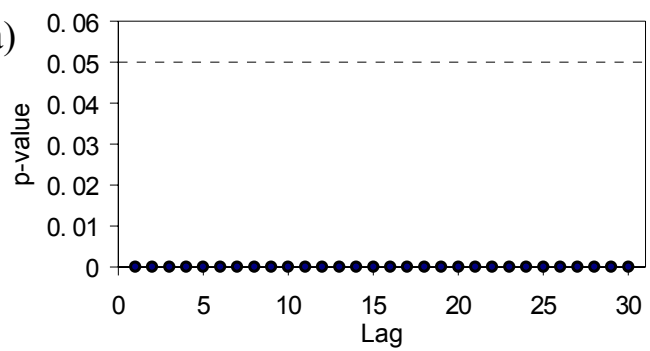

(b)

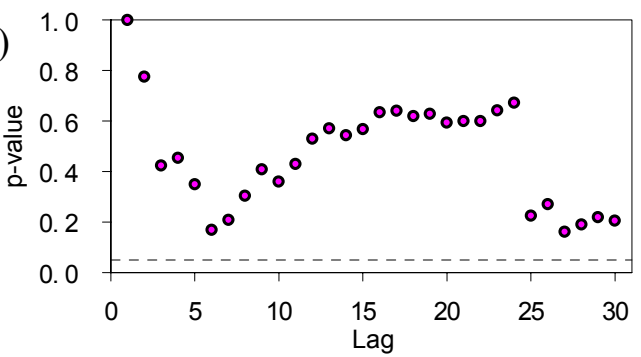

Fig. 13. Engle's LM test for seasonally standardized residuals from (a) the ARMA(20,1) model for daily flow and (b) from the AR(4) model for monthly flow.

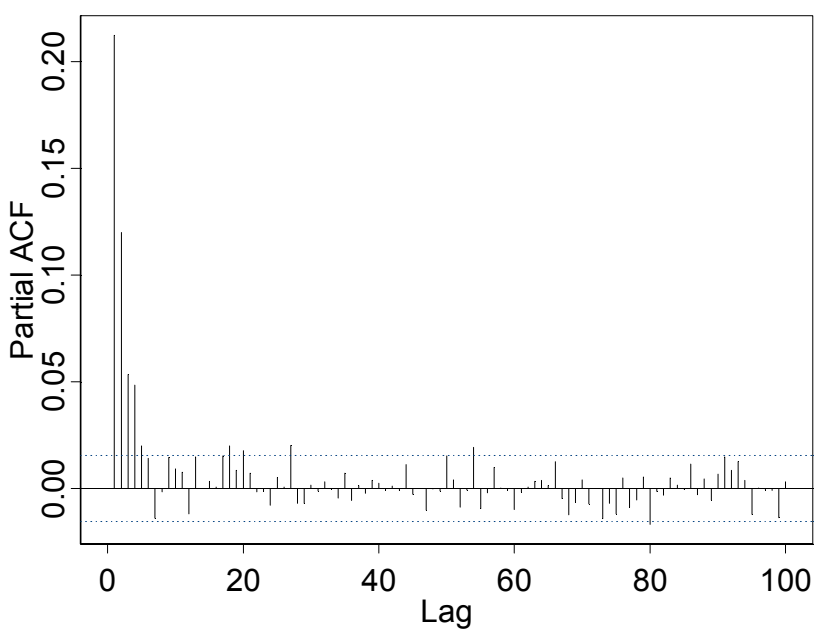

Fig. 14. PACF of the squared seasonally starndardized residual series from $\operatorname{ARMA}(20,1)$ for daily flow.

\section{Modelling the daily steamflow with ARMA-GARCH error model}

\subsection{Model building}

Weiss (1984) proposed ARMA models with ARCH errors. This approach is adopted and extended by many researchers for modelling economic time series (e.g. Hauser and Kunst, 1998; Karanasos, 2001). In the field of geo-sciences, Tol (1996) fitted a GARCH model for the conditional variance and the conditional standard deviation, in conjunction with an AR(2) model for the mean, to model daily mean temperature. In this paper, we propose to use ARMA-GARCH error (or, for notation convenience, called ARMA-GARCH) model for modelling daily streamflow processes.

The ARMA-GARCH model may be interpreted as a combination of an ARMA model which is used to model mean behaviour, and an ARCH model which is used to model the ARCH effect in the residual series from the ARMA model. The ARMA model has the form as in Eq. (4). The
$\operatorname{GARCH}(p, q)$ model has the form (Bollerslev, 1986)

$$
\left\{\begin{array}{c}
\varepsilon_{t} \mid \psi_{t-1} \sim N\left(0, h_{t}\right) \\
h_{t}=\alpha_{0}+\sum_{i=1}^{q} \alpha_{i} \varepsilon_{t-i}^{2}+\sum_{i=1}^{p} \beta_{i} h_{t-i},
\end{array}\right.
$$

where, $\varepsilon_{t}$ denotes a real-valued discrete-time stochastic process, and $\psi_{t}$ the available information set, $p \geq 0, q>0$, $\alpha_{0}>0, \alpha_{i} \geq 0, \beta_{i} \geq 0$. When $p=0$, the $\operatorname{GARCH}(p, q)$ model reduces to the $\operatorname{ARCH}(q)$ model. Under the $\operatorname{GARCH}(p, q)$ model, the conditional variance of $\varepsilon_{t}, h_{t}$, depends on the squared residuals in the previous $q$ time steps, and the conditional variance in the previous $p$ time steps. Since GARCH models can be treated as ARMA models for squared residuals, the order of GARCH can be determined with the method for selecting the order of ARMA models, and traditional model selection criteria, such as Akaike information criterion (AIC) and Bayesian information criterion (BIC), can also be used for selecting models. The unknown model parameters $\alpha_{i}(i=0, \cdots \cdot, q)$ and $\beta_{j}(j=1, \cdot \quad \cdot \quad \cdot, p)$ can be estimated using (conditional) maximum likelihood estimation (MLE). Estimates of the conditional standard deviation $h_{t}^{1 / 2}$ are also obtained as a side product with the MLE method.

When there is obvious seasonality present in the residuals (as in the case of daily streamflow at Tangnaihai), to preserve the seasonal variances in the residuals, instead of fitting the $\mathrm{ARCH}$ model to the residual series directly, we fit the $\mathrm{ARCH}$ model to the seasonally standardized residual series, which is obtained by dividing the residual series by seasonal standard deviations (i.e. daily standard deviations for daily flow). Therefore, the general ARMA-GARCH model with seasonal standard deviations we propose here has the following form

$$
\left\{\begin{array}{l}
\phi(B) x_{t}=\theta(B) \varepsilon_{t} \\
\varepsilon_{t}=\sigma_{s} z_{t}, \quad z_{t} \sim N\left(0, h_{t}\right) \\
h_{t}=\alpha_{0}+\sum_{i=1}^{q} \alpha_{i} z_{t-i}^{2}+\sum_{i=1}^{p} \beta_{i} h_{t-i}
\end{array},\right.
$$

where $\sigma_{s}$ is the seasonal standard deviation of $\varepsilon_{t}, s$ is the season number depending on which season the time $t$ belongs to. For daily series, $s$ ranges from 1 to 366 . Other notations are the same as in Eqs. (4) and (6).

The model building procedure proceeds in the following steps: 



Fig. 15. A segment of (a) the seasonally standardized residuals from ARMA(20,1) and (b) its corresponding conditional standard deviation sequence estimated with the $\mathrm{ARCH}(21)$ model.
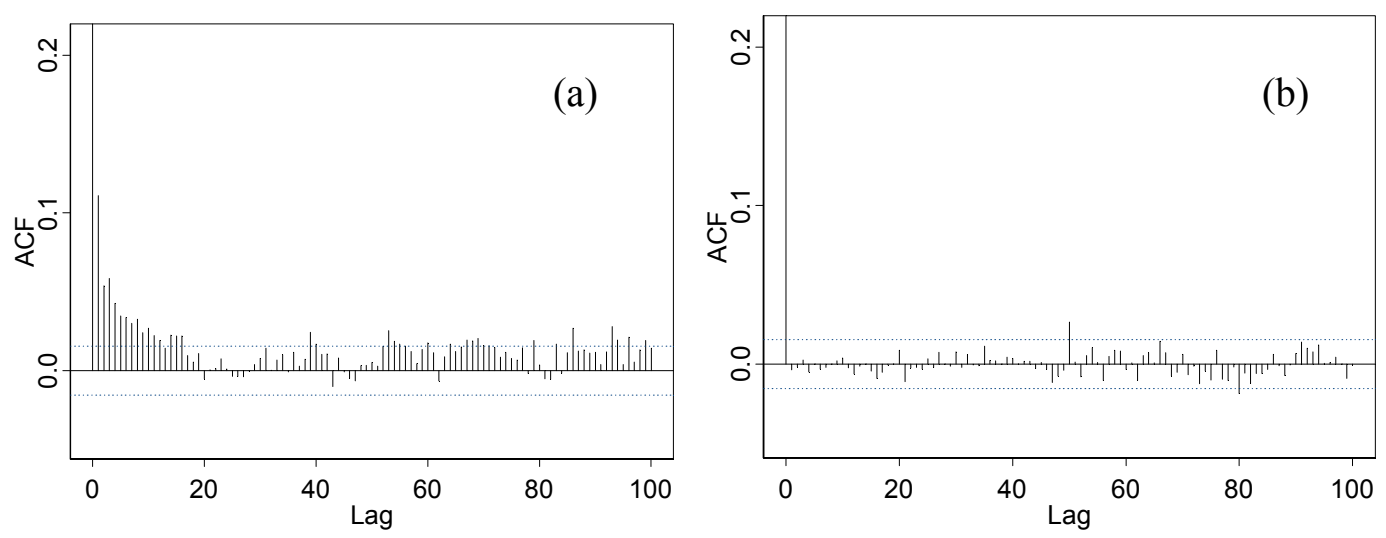

Fig. 16. ACFs of (a) the standardized residuals and (b) squared standardized residuals from the ARMA(20,1)-ARCH(21) model. The standardization is accomplished by dividing the seasonally standardized residuals from ARMA $(20,1)$ by the conditional standard deviation estimated with $\mathrm{ARCH}(21)$.

1. Logarithmize and deseasonalize the original flow series;

2. Fit an ARMA model to the logarithmized and deseasonalized flow series;

3. Calculate seasonal standard deviations of the residuals obtained from ARMA model, and seasonally standardize the residuals with the first 8 Fourier harmonics of the seasonal standard deviations;

4. Fit a GARCH model to the seasonally standardized residual series.

For forecasting and simulation, inverse transformation (including logarithmization and deseasonalization) is needed. When forecasting, the ARMA part of the ARMA-GARCH model forecasts future mean values of the underlying time series following the traditional approach for ARMA prediction, whereas the GARCH part gives forecasts of future volatility, especially over short horizons.

Following the above-mentioned steps, a preliminary ARMA-GARCH model is fitted to the daily streamflow series at Tangnaihai. The ACF and PACF structure of the squared seasonally standardized residuals are shown in Fig. 12a and Fig. 14, respectively. According to the AIC, as well as the ACF and PACF structure, a $\operatorname{GARCH}(0,21)$ model, i.e. $\operatorname{ARCH}(21)$ model, which has the smallest AIC value is selected. Therefore, the prelimilary ARMA-GARCH model fitted to the daily streamflow series at Tangnaihai is composed of an ARMA(20,1) model and an ARCH(21) model. The model is constructed with statistics software S-Plus (Zivot and Wang, 2003).

\subsection{Model diagnostic and modification}

If the ARMA-GARCH model is successful in modelling the serial correlation structure in the conditional mean and conditional variance, then there should be no autocorrelation left in both the residuals and the squared residuals standardized by the estimated conditional standard deviation.

A segment of the seasonally standardized residual series from the $\operatorname{ARMA}(20,1)$ model and its corresponding conditional standard deviation sequence estimated with the $\mathrm{ARCH}(21)$ model are shown in Figs. 15a and 15b. We standardize the seasonally standardized residual series from 

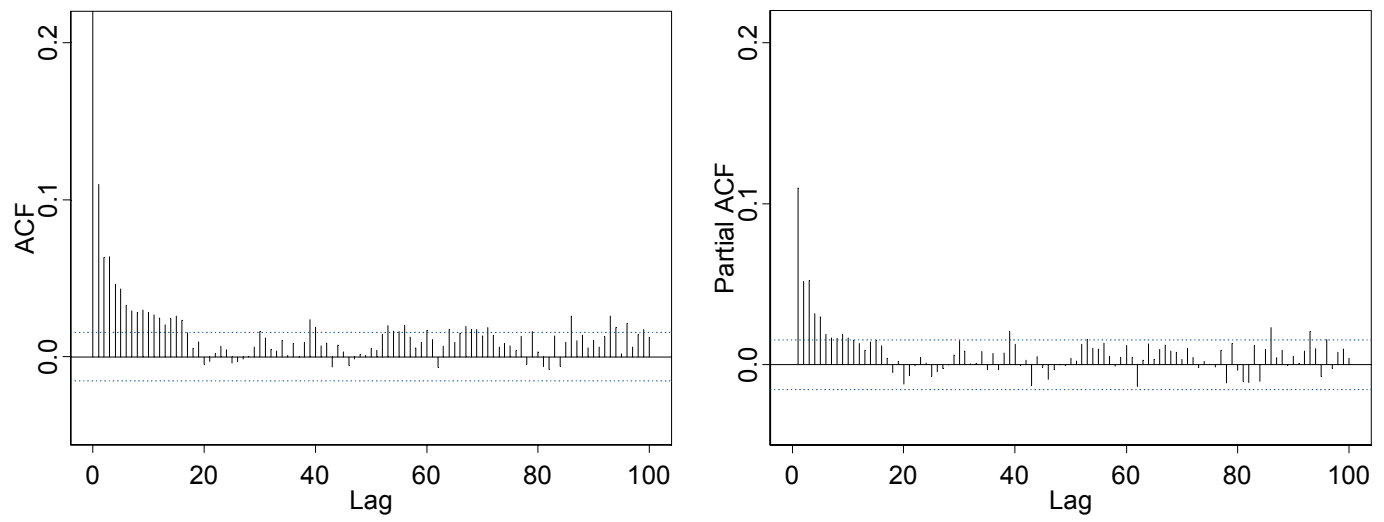

Fig. 17. ACF and PACF of seasonally standardized residuals from the ARMA(20,1) model.
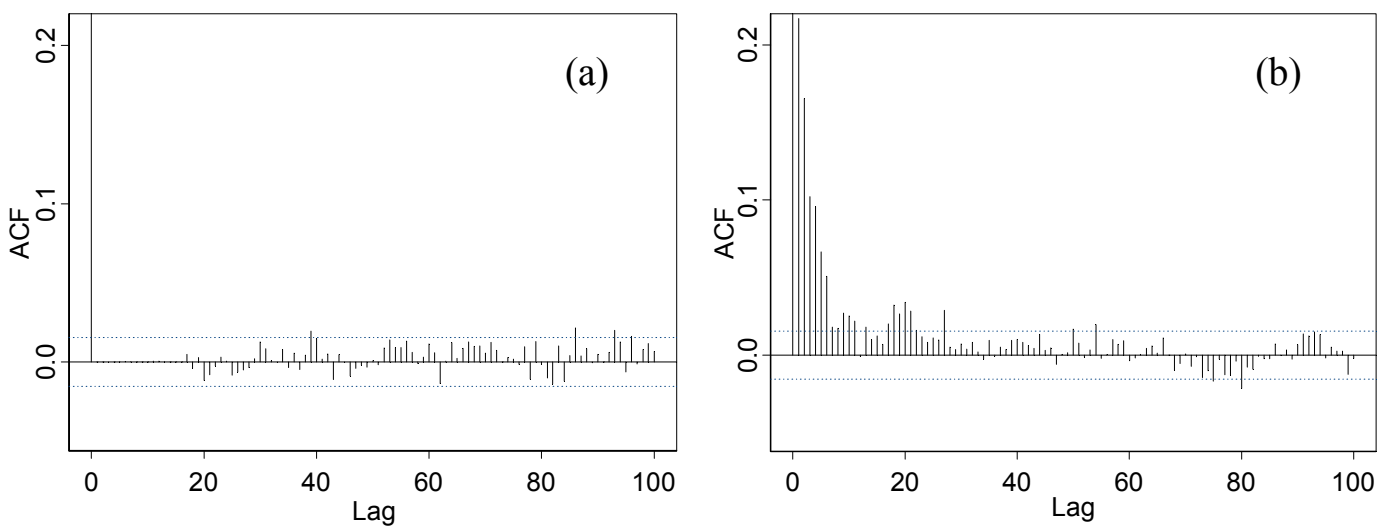

Fig. 18. ACFs of (a) the second-residuals and (b) the squared second-residuals from the ARMA(20,1)-AR(16) model.

the $\operatorname{ARMA}(20,1)$ model by dividing it by the estimated conditional standard deviation sequence. The autocorrelations of the standardized residuals and squared standardized residuals are plotted in Fig. 16. It is shown that although there is no autocorrelation left in the squared standardized residuals, which means that the $\mathrm{ARCH}$ effect has been removed (Fig. 16b), however, in the non-squared standardized residuals of daily flow significant autocorrelation remains (Fig. 16a).

Because the GARCH model is designed to deal with the conditional variance behavior, rather than mean behavior, the autocorrelation in the non-squared residual series must arise from the seasonally standardized residuals obtained in step 3 of the ARMA-GARCH model building procedure. Therefore we revisit the seasonally standardized residuals. It is found that although the residuals from the $\operatorname{ARMA}(20,1)$ model present no obvious autocorrelation as shown in Fig. 6a, weak but significant autocorrelations in the residuals are revealed after the residuals are seasonally standardized, as shown by the ACF and PACF in Fig. 17. We refer to this weak autocorrelation as the hidden weak autocorrelation.

The mechanism underlying such weak autocorrelation is not clear yet. Similar phenomena are also found for some other daily streamflow processes (such as the daily stream- flow of the Umpqua River near Elkton and the Wisconsin River near Wisconsin Dells, available on the USGS website http://water.usgs.gov/waterwatch), which have strong seasonality in the ACF structures of their original series, as well as their residual series. To handle the problem of the weak correlations, an additional ARMA model is needed to model the mean behaviour in the seasonally standardized residuals, and a GARCH is then fitted to the residuals from this additional ARMA model. Therefore, we obtain an extended version of the model in Eq. (7) as

$$
\left\{\begin{array}{l}
\phi(B) x_{t}=\theta(B) \varepsilon_{t} \\
\varepsilon_{t}=\sigma_{s} y_{t} \\
\phi^{\prime}(B) y_{t}=\theta^{\prime}(B) z_{t}, \quad z_{t} \sim N\left(0, h_{t}\right), \\
h_{t}=\alpha_{0}+\sum_{i=1}^{q} \alpha_{i} z_{t-i}^{2}+\sum_{i=1}^{p} \beta_{i} h_{t-i}
\end{array}\right.
$$

where $y_{t}$ is the seasonally standardized residuals from the first ARMA model, $z_{t}$ is the residuals (for notation convenience, we call it second-residuals) from the second ARMA model fitted to $y_{t}$.

An AR(16) model, whose autoregressive order is chosen according to AIC, is fitted to the seasonally standardized residuals from the ARMA $(20,1)$ model of the daily flow series at Tangnaihai, and we obtain a second-residual se- 

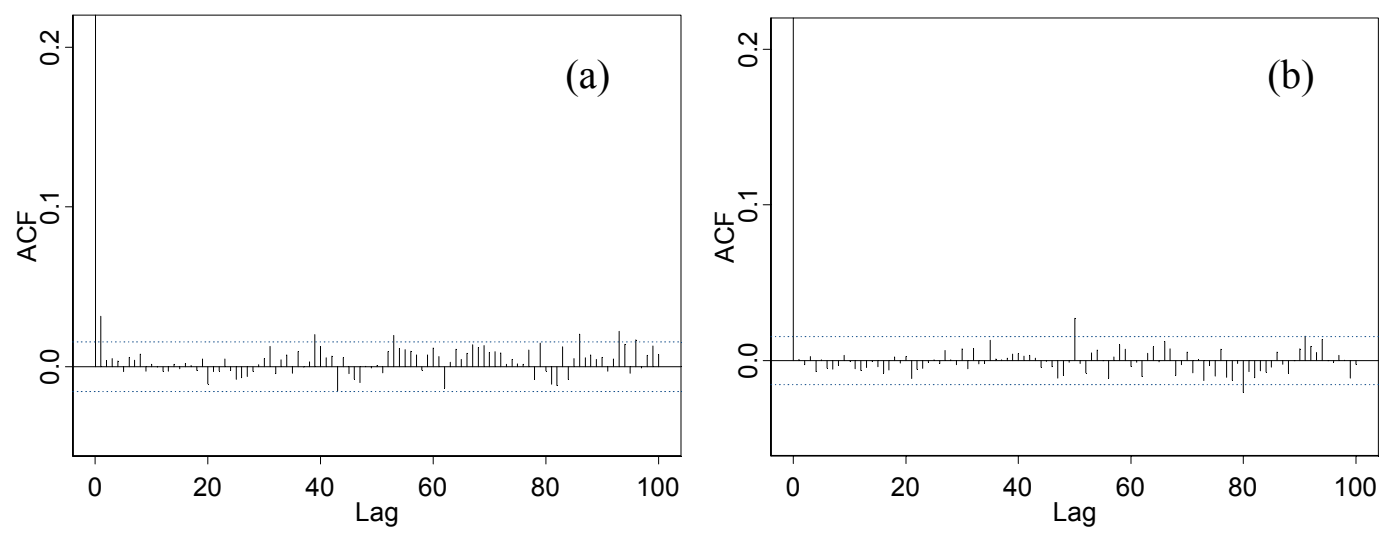

Fig. 19. ACFs of (a) the standardized second-residuals and (b) the squared standardized second-residuals from the ARMA(20,1)-AR(16)$\operatorname{ARCH}(21)$ model. The second-residuals are obtained from AR(16) fitted to the seasonally standardized residuals from ARMA(20,1).

ries from this $\mathrm{AR}(16)$ model. The autocorrelations of the second-residual series and the squared second-residual series from the $\operatorname{ARMA}(20,1)-\operatorname{AR}(16)$ combined model are shown in Fig. 18. From visual inspection, we find that no autocorrelation is left in the second-residual series, but there is strong autocorrelation in the squared second-residual series which indicates the existence of an ARCH effect.

Because the squared second-residual series has similar ACF and PACF stucture to the seasonally standardized residuals from the $\operatorname{ARMA}(21,0)$ model, the same structure of the GARCH model, i.e. an $\mathrm{ARCH}(21)$ model, is fitted to the second-residual series. Therefore, the ultimate ARMAGARCH model fitted to the daily streamflow at Tangnaihai is $\operatorname{ARMA}(20,1)-\operatorname{AR}(16)-\operatorname{ARCH}(21)$, composed of an $\operatorname{ARMA}(20,1)$ model fitted to logarithmized and deseasonalized series, an AR(16) model fitted to the seasonally standardized residuals from the $\operatorname{ARMA}(20,1)$ model, and an $\operatorname{ARCH}(21)$ model fitted to the second-residuals from the AR(16) model.

We standardize the second-residual series with the conditional standard deviation sequence obtained with the ARCH(21) model. The autocorrelations of the standardized second-residuals and the squared standardized secondresiduals are shown in Fig. 19. Compared with Fig. 16, the autocorrelations are basically removed for both the squared and non-squared series, although the autocorrelation at lag 1 of the standardized second-residuals slightly exceeds the 5\% significance level. The McLeod-Li test and the LMtest (shown in Fig. 20) for standardized second-residuals also confirm that the ARCH(21) model fits the second-residual series well. The small lag-1 autocorrelation in the standardized second-residual series (shown in Fig. 19) is a hidden autocorrelation covered by conditional heteroskedasticity. This autocorrelation can be further modeled with another AR model, but because the autocorrelation is very small, it could be neglected.

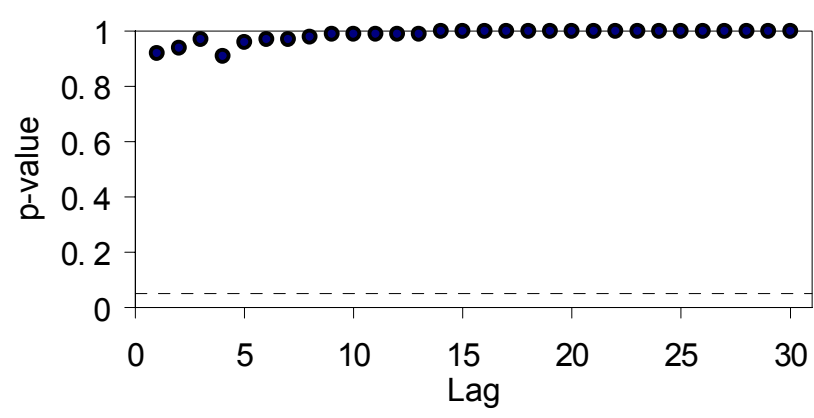

Fig. 20. Engle's LM test for the standardized second-residuals from the ARMA(20,1)-AR(16)-ARCH(21) model.

\section{Conclusions}

The nonlinear mechanism conditional heteroskedasticity in hydrologic processes has not received much attention in the literature so far. Modelling data with time varying conditional variance could be attempted in various ways, including nonparametric and semi-parametric approaches (see Lall, 1995; Sankarasubramanian and Lall, 2003). A parametric approach with ARCH model is proposed in this paper to describe the conditional variance behavior. ARCH-type models which originate from econometrics can provide accurate forecasts of variances. As a consequence, they can be applied to such diverse fields as water management risk analysis, prediction uncertainty analysis and streamflow series simulation.

The existence of conditional heteroskedasticity is verified in the residual series from linear models fitted to the daily and monthly streamflow processes of the upper Yellow River with the McLeod-Li test and the Engle's Lagrange Multiplier test. It is shown that the ARCH effect is fully caused by seasonal variation in variance for monthly flow, but seasonal variation in variance only partly explains the ARCH effect for daily streamflow. Among three types of conventional seasonal time series model (i.e. SARIMA, deseasonalized ARMA and PARMA), none of them is efficient enough to describe the ARCH effect for daily flow, although the 
PARMA model is enough for monthly flow by considering season-dependent variances. Therefore, to fully capture the $\mathrm{ARCH}$ effect, as well as the seasonal variances inspected in the residuals from linear ARMA models fitted to the daily flow series, the ARMA-GARCH error model with seasonal standard deviations is proposed. The ARMA-GARCH model is basically a combination of an ARMA model which is used to model mean behaviour, and a GARCH model to model the ARCH effect in the residuals from the ARMA model. To preserve the seasonal variation in variance in the residuals, the ARCH model is not fitted to the residual series directly, but to the seasonally standardized residuals. Therefore, an important feature of the ARMA-GARCH model is that the unconditional seasonal variance of the process is seasonally constant but the conditional variance is not. To resolve the problem of the weak hidden autocorrelation revealed after the residuals are seasonally standarized, the ARMA-GARCH model is extended by applying an additional ARMA model to model the mean behaviour in the seasonally standardized residual series. With such a modified ARMA-GARCH model, the daily streamflow series is well-fitted.

Because the ARCH effect in daily streamflow mainly arises from daily variations in temperature and precipitation, and given that we have reasonably good skill in predicting weather two to three days in advance (for example, see http://weather.gov/rivers_tab.php), the use in developing an ARMA-GARCH model would be limited. However, because (1) on the one hand, the relationship between runoff and rainfall and temperature is hard to capture precisely by any model so far; (2) on the other hand, usually there are not enough rainfall data available to fully capture the rainfall spatial pattern, especially for remote areas, such as Tibet Plateau, and (3) the accuracy of the weather forecasts for these areas are very limited, the ARCH effect cannot be fully removed even after limited rainfall data and temperature data are included in the model. Therefore, the ARMA-GARCH model would be a very useful addition in terms of statistical modelling of daily streamflow processes for the hydrological community.

Acknowledgements. We are very grateful to I. McLeod and an anonymous reviewer. Their comments, especially the detailed comments from the anonymous reviewer, are very helpful to improve the paper considerably.

Edited by: B. Sivakumar

Reviewed by: I. McLeaod and another referee

\section{References}

Bollerslev, T.: Generalized Autoregressive Conditional Heteroscedasticity, Journal of Econometrics, 31, 307-327, 1986.

Box, G. E. P. and Jenkins, J. M.: Time series analysis: Forecasting and control, San Francisco, Holden-Day, 1976.

Brock, W. A., Dechert, W. D., Scheinkman, J. A., and LeBaron, B.: A test for independence based on the correlation dimension, Econ. Rev., 15, 3, 197-235, 1996.

Engle, R.: Autoregressive conditional heteroscedasticity with estimates of the variance of UK inflation, Econometrica, 50, 9871008, 1982.

Granger, C. W. J. and Andersen, A. P.: An introduction to bilinear time series models, Göttingen, Vandenhoeck and Ruprecht, 1978.

Hipel, K. W. and McLeod, A. I.: Time series modelling of water resources and environmental systems, Elsvier, Amsterdam, 1994.

Karanasos, M.: Prediction in ARMA models with GARCH-inmean effects, Journal of Time Series Analysis, 22, 555-78, 2001.

Lall, U.: Recent advance in nonparametric function estimation hydrologic application, Reviews of Geophysics, 33, 1093-1102, 1995.

Livina, V., Ashkenazy, Y., Kizner, Z., Strygin, V., Bunde, A., and Havlin, S.: A stochastic model of river discharge fluctuations, Physica A, 330, 283-290, 2003.

Ljung, G. M. and Box, G. E. P.: On a measure of lack of fit in time series models, Biometrika, 65, 297-303, 1978.

McLeod, A. I. and Li, W. K.: Diagnostic checking ARMA time series models using squared residual autocorrelations, Journal of Time Series Analysis, 4, 269-273, 1983.

Miller, R. B.: Book review on "An Introduction to Bilinear Time Series Models" by Granger, C. W. and Andersen, A. P., J. Amer. Statist. Ass., 74, 927, 1979.

Hauser, M. A. and Kunst, R. M.: Fractionally Integrated Models With ARCH Errors: With an Application to the Swiss 1-Month Euromarket Interest Rate, Review of Quantitative Finance and Accounting, 10, 95-113, 1998.

Salas, J. D.: Analysis and modelling of hydrologic time series. In: Handbook of Hydrology, edited by Maidment, D. R., McGrawHill, 19.1-19.72, 1993.

Sankarasubramanian, A. and Lall, U.: Flood quantiles in a changing climate: Seasonal forecasts and causal relations, Water Resources Research, 39, 5, Art. No. 1134, 2003.

Tol, R. J. S.: Autoregressive conditional heteroscedasticity in daily temperature measurements, Environmetrics, 7, 67-75, 1996.

Wang, W., Van Gelder, P. H. A. J. M., and Vrijling, J. K.: Periodic autoregressive models applied to daily streamflow. In: 6th International Conference on Hydroinformatics, edited by Liong, S. Y., Phoon, K. K. and Babovic, V., Singapore, World Scientific, 1334-1341, 2004.

Weiss, A. A.: ARMA models with ARCH errors, Journal of Time Series Analysis, 5, 129-143, 1984.

Zivot, E. and Wang, J.: Modelling Financial Time Series with SPLUS, New York, Springer, 2003. 